\title{
PRKAR1B-related neurodegenerative dementia with intermediate filaments
}

INSERM

\section{Source}

INSERM. (1999). Orphanet: an online rare disease and orphan drug data base. PRKAR1Brelated neurodegenerative dementia with intermediate filaments. ORPHA:412066

PRKAR1B-related neurodeg enerative dementia with intermediate filaments is a rare, genetic neurodegenerative disease characterized by dementia and mild parkinsonism with poor levodopa response. Presenting clinical manifestations are memory problems, short attention span, disorientation, language impairment, rigidity, bradykinesia, postural instability and behavioral changes, including apathy, anxiety and delusions. 